\title{
GESTIÓN AMBIENTAL ARTICULADA, SOSTENIBLE $Y$ EFECTIVA EN COSTA RICA ${ }^{1}$
}

\author{
ENVIRONMENTAL MANAGEMENT IN COSTA RICA: \\ ARTICULATED, SUSTAINABLE AND EFFECTIVE
}

\author{
Leda Peralta Quesada* \\ Mariana Solano Umanzor**
}

RESUMEN

Durante la Administración Arias Sánchez el tema ambiental se ha visto sumamente afectado por políticas que atentan contra su preservación y mantenimiento. Específicamente, el tema del ordenamiento territorial representa uno de los problemas socioambientales más serios y persistentes; viéndose afectado no sólo por la deficiente legislación y débil implementación, sino por la grave descoordinación institucional que se ha convertido en un eje transversal presente en todos los niveles de organización gubernamental en el país.

PALABRAS CLAVE: COSTA RICA * RECURSOS NATURALES * DETERIORO AMBIENTAL * POLÍTICA GUBERNAMENTAL * PLANIFICACIÓN URBANA * LEGISLACIÓN

ABSTRACT

During the Arias Sanchez Administration the environmental agenda has been seriously affected by policies that threaten its preservation and maintenance. Specifically, the land use has become one of the more serious and persistent socioenvironmental problem; being affected not only by deficient legislation and weak implementation, but also by the severe lack of coordination between institutions that has turn into a transversal axis present in every level of the governmental organization in the country.

KEY WORDS: COSTA RICA * NATURAL RESOURCES * ENVIRONMENTAL DEGRADATION * GOVERNMENT POLICY $*$ URBAN PLANNING $*$ LEGISLATION

1 Este artículo forma parte de las investigaciones presentadas en el concurso Voces Nuevas en Ciencias Sociales, del Instituto de Investigaciones Sociales de la Universidad de Costa Rica. Abril de 2010 .

\footnotetext{
* Ministerio de Planificación Nacional y Política Económica, Costa Rica. ledaperalta@gmail.com

** Programa Estado de la Nación, Costa Rica. mariana.umanzor.solano@gmail.com
} 


\section{INTRODUCCIÓN}

La Administración Arias Sánchez, así como las últimas administraciones han recalcado durante campaña su interés en mejorar, conservar y mantener un medioambiente equilibrado, esto tiene especial sentido y relevancia por el hecho de que Costa Rica se ha convertido en un destino turístico natural por excelencia en la región americana, el cual ha establecido una marca país de reconocimiento y valor internacional.

Sin embargo, más allá de que los indicadores y cumplimiento de metas del sector ambiente, energía y telecomunicaciones son bastante satisfactorios, existe un descontento generalizado entre los ambientalistas y conservacionistas sobre la actuación del gobierno durante estos cuatro años.

En el Plan Nacional de Desarrollo (PND) 2006-2010 se incluyen ocho metas nacionales, sin embargo, los temas medioambiental y de ordenamiento territorial no se encuentran dentro de ellas. La meta 6, que se refiere a "fortalecer las instituciones públicas y ordenar las prioridades del Estado" no contempla al ambiente ni ordenamiento como prioridades, sino que se centra en disminuir la tramitología.

Las cifras ofrecidas por el Ministerio de Planificación Nacional y Política Económica (MIDEPLAN) son satisfactorias, no obstante, es necesario analizar otras fuentes de información para poder tener un panorama más amplio sobre el estado actual del sector ambiental, y sobre la labor que desempeñaron los Ministros de Ambiente, Energía y Telecomunicaciones durante el período en estudio.

\section{HUELLA ECOLÓGICA}

Durante las últimas Administraciones, se ha tratado el tema medioambiental de una manera tangencial $y$ desde una perspectiva económica. Costa Rica cuenta con recursos naturales muy importantes en relación con su pequeño territorio, sin embargo, de seguir al ritmo de consumo actual, estos recursos estarán cada vez más en riesgo.
Del estudio del PND se desprende que, de las metas específicas del sector medioambiental, 15 se refieren a diseño de planes, y sólo doce a implementación o acciones directas y concretas. De esos 15 planes o informes, 14 presentan "avance satisfactorio" y sólo uno con "atraso crítico". En cuanto a la implementación de planes y acciones, el panorama es mixto y pareciera que el problema se presenta a la hora de llevar a cabo las acciones concretas para la inmensa variedad de problemas existentes en el país.

El XIII Informe del Estado de la Nación describe claramente este problema:

... En el balance es claro que aun las acciones públicas realizadas, pese a ser necesarias, no son suficientes para la adecuada protección del ambiente (...) Y también porque parte de los avances se limita a la formulación de estrategias, políticas y planes de respuesta; los problemas de cumplimiento $y$ de capacidad de regulación, así como la gravedad de las situaciones precedentes, hacen que esas iniciativas no puedan ser evaluadas sino hasta dentro de varios años.

En el tema medio ambiental, lo más grave es que los problemas permanecen sin ser resueltos. Con base en el XIII, XIV y XV Informes del Estado de la Nación, así como resultado de consultas en periódicos nacionales, se constató que los principales problemas que persisten en el país son: el recurso hídrico, el ordenamiento territorial, la crisis energética, el cambio climático, la minería, la necesidad de energías alternativas, la depredación de los recursos marinos, el caos en la delimitación costera, el riesgo de las Áreas Silvestres Protegidas y la descoordinación interinstitucional. Siendo este último un tema transversal que afecta prácticamente todas las esferas del ámbito político nacional, a continuación se esbozan brevemente tres casos que ejemplifican claramente la orientación de la política ambiental del período 2006-2010.

En el tema del recurso hídrico por ejemplo no ha sido aprobado en la Asamblea Legislativa el expediente 14585 correspondiente a la Ley del Recurso Hídrico, ignorándose 
importantes llamados de atención, como el conflicto sucedido en Sardinal en 2008, en torno a la ampliación de un acueducto. Posteriormente a que la Sala Constitucional paralizara el proyecto por falta de certeza técnica y omisión en la participación ciudadana, un grupo de investigadores de la Universidad de Costa Rica emitió un informe sobre este conflicto, concluyéndose que ha habido una gestión deficiente del recurso. Según el periódico El Financiero 2010:

...[el informe] insta a entidades gubernamentales, al AyA y Municipalidad de Carrillo a abrir órganos de procedimientos para estudiar las actuaciones y omisiones de los funcionarios que tomaron decisiones en torno al tema. También sugiere a estas instancias iniciar procesos de lesividad por el otorgamiento de permisos $y$ patentes dados ilegalmente en torno al acceso de agua (...). Se actuó irresponsablemente por parte de AyA $y$ del Minaet.

En cuanto a los recursos marinos, cabe mencionar que el área marina de Costa Rica es diez veces mayor que su territorio, por lo que sus recursos marinos son vastos y diversos. Sin embargo, se ha avocado a la protección de sus recursos naturales terrestres, dejando de lado los recursos marinos, de manera que el crecimiento del sector pesquero ha sido desordenado e intensivo, por lo que las diferentes especies están en peligro.

Además, producto de la deficiente legislación, vacíos y falta de implementación en los muelles privados se dan situaciones irregulares que el Estado no tiene la capacidad de controlar, tales como tráfico de drogas, pesca ilegal, sobre pesca, contrabando y aleteo. Por otra parte, cabe mencionar dos puntos importantes. De acuerdo con (Jiménez, La Nación 2010) la ley de pesca publicada hace cinco años sigue sin reglamento y el Instituto Costarricense de Pesca y Acuicultura (INCOPESCA) no cuenta con los recursos para hacer frente a este importante reto. Y segundo, “(...) INCOPESCA es políticamente dependiente del grupo que regula: buena parte de su Junta Directiva está compuesta por los representantes de los pescadores, por lo que su independencia como regulador de la pesca está seriamente comprometida".

Finalmente, el estrepitoso desarrollo inmobiliario del Pacífico costarricense (específicamente en la provincia de Guanacaste) ha evidenciado la separación existente entre el crecimiento económico y el desarrollo sostenible. Algunos ejemplos de los daños generados por este crecimiento descontrolado y no planificado en las costas del país, son las dificultades que han afrontado varias comunidades en la provincia de Guanacaste, debido a la escasez de agua, ante el uso excesivo de los complejos hoteleros en la irrigación de las canchas de golf.

Además en cuanto a creación de nuevos parques nacionales, esta ha sido una de las pocas administraciones que no crea un nuevo parque, un ejemplo reciente de esta situación es la región del Parque Nacional Marino Las Baulas (PNMLB) donde se ha dado un crecimiento inmobiliario impresionante, de manera que se ha tratado de abrir al desarrollo inmobiliario, bajando su categoría de Parque Nacional a Refugio de Vida Silvestre. Esto afectaría no sólo la categoría del área, sino que agravaría aún más los daños a la anidación de tortugas baulas en uno de los lugares más importantes del mundo. En cuanto al Refugio Gandoca-Manzanillo, el Decreto Ejecutivo 34043-MINAE varía sus límites. En dicha área se protegen humedales y arrecifes, así como especies en peligro de extinción como los manatíes, las tortugas baula, verde y carey.

De acuerdo con un artículo del Semanario Universidad (Araya, 2010):

Como consecuencia del crecimiento desordenado en las construcciones debido al turismo y sus actividades complementarias, la ZMT está mostrando indicios de degradación de manglares y desaparición de humedales costeros, pérdida y contaminación de sistemas de dunas de playa $y$ otras plataformas abrasivas por la producción de desechos sólidos y la evacuación de las aguas negras, deterioro del paisaje, alteración de cauces, salinización de los mantos acuíferos y los manantiales, $y$ un excesivo consumo de agua potable. 
SÍNTESIS DE LA SITUACIÓN AMBIENTAL EN COSTA RICA

Los ejemplos de problemáticas ambientales abundan, $y$ todos indican que Costa Rica, pese a su marca país verde, no está haciendo los esfuerzos necesarios para fortalecer las instituciones $y$ mejorar las acciones en torno al tema ambiental. Más allá de que mucha de la normativa puede ser mejorada, es necesario reconocer que sí existe legislación en casi todos los ámbitos, sin embargo, existe un profundo problema cuando se trata de la aplicación de las normas. Por otra parte hay una seria descoordinación inter e intrainstitucional. Además, se ha demostrado la complicidad entre algunas instituciones públicas y sectores privados para poder llevar adelante prácticas ilegales y depredadoras para el ambiente.

En resumen a partir del XIII Informe del Estado de la Nación, la Contraloría General de la República ha planteado que:

...El sector ambiental se encuentra desarticulado en algunas áreas, existe diversidad de leyes $y$ funciones; hay traslado y dispersión de funciones; vacíos de competencias; ineficiente coordinación y gestión institucional; $y$ en algunos casos inviabilidad de aplicar normas sancionatorias en materia de responsabilidad administrativa $y$ civil $^{2}$.

Los problemas ambientales son ampliamente conocidos por todos e ignorados por algunos. En el Manifiesto Público "En defensa de la naturaleza", de la Revista Ambientico se identifican claramente los principales problemas, así como el camino que el país debería seguir. Se hace un llamado a la opinión pública nacional e internacional sobre las contradicciones existentes entre el discurso político de la Administración Arias Sánchez, y la falta de políticas públicas para enfrentar los riesgos que se corren.

2 Castro, R. "Actividades productivas y legislación ambiental”. Capítulo Armonía con el Ambiente. XIII Informe del Estado de la Nación. Octubre 2007.
Más recientemente la Organización Llamado Urgente por el País, en comunicado de prensa del 2010 realizó un ejercicio similar, y también llegan a la conclusión de que existe un desfase entre el discurso ambiental de la Administración y las acciones realizadas. De manera que se puede resumir el documento de la siguiente manera:

Con una contundente línea seguida desde sus inicios: el beneficio para una minoría en detrimento de una mayoría. El discurso ambiental, en foros mundiales, basándose en una larga trayectoria del país y su histórico compromiso con el ambiente, no tuvo ninguna consistencia a lo interno del país, provocando un profundo retroceso y generando daños posiblemente irreversibles que pagarán no solo las generaciones actuales, sino también las futuras.

Sin embargo, merece la pena reconocer los avances en esta materia. De acuerdo con el XIV Informe del Estado de la Nación, principalmente se relaciona con la conservación y el patrimonio natural:

La expansión durante varias décadas de su área protegida y los altos índices de representación de biodiversidad y ecosistemas bajo resguardo han contribuido a generar la percepción de que Costa Rica tiene bases sólidas en este campo. Sin embargo, la información disponible señala la importancia de entender estos logros como vulnerables, susceptibles de los cambios en las políticas, recursos o voluntades de muchos actores. Esto se debe, en primer lugar, al peso de los desafíos que se encuentran fuera de las áreas protegidas y que las afectan; en segundo, al hecho comprobado de que el establecimiento formal de esas áreas no garantiza su sostenibilidad.

Por otra parte, se han dado importantes avances en la recuperación de cobertura forestal del país. Sin embargo, nuevamente el Estado de la Nación en su informe XIII advierte sobre la vulnerabilidad de estos logros, debido a que no 
se garantiza la sostenibilidad a largo plazo, la recuperación se da en zonas sin protección, las zonas recuperadas son susceptibles a ser deforestadas nuevamente, $y$ que de las áreas cubiertas de bosque sólo un 44\% tiene algún grado de protección. Por lo tanto es necesario trabajar en esos temas para garantizar que los logros en materia de patrimonio natural se mantengan.

Hasta el momento se analizaron brevemente algunos de los problemas medioambientales más serios y constantes del país, un tema mencionado fue el ordenamiento territorial que se trata de manera más extensa a continuación. Para ello, se estudian específicamente las implicaciones del Decreto Ejecutivo 35748-MP-MINAET-MIVAH como mecanismo para confirmar las causas generales que han provocado que la Administración Arias Sánchez deje mucho que desear al final de su gestión.

\section{ALCANCES DEL DECRETO EJECUTIVO 35748-MP-MINAET-MIVAH}

El pasado 19 de febrero de 2010 la Administración Arias Sánchez promulgó el Decreto Ejecutivo 35748-MP-MINAET-MIVAH, el cual adiciona el inciso 3.7 al artículo 3 del Anexo 1 del Plan Regional de Desarrollo Urbano "Gran Área Metropolitana" lo siguiente:

Aprobada la viabilidad ambiental, en los cantones que no cuentan con Plan Regulador vigente $y$ que en su territorio se definan zonas de moderada 0 alta fragilidad ambiental, respetando en un todo el Protocolo Ambiental autorizado por Secretaría Técnica Nacional Ambiental (SETENA), permite a las fincas, por cuyos terrenos se definió el límite de contención urbana, o que estén próximos con este a no más de cien metros (100 metros), exceder el uso urbano dentro del área de protección en un porcentaje no mayor del 50\% del área total de la finca, o hasta doscientos metros (200 metros) del límite, cualquiera que fuese menor, obligándose cada Gobierno Local, a aplicar esta normativa en los certificados de usos de suelo que otorgue.
A grandes rasgos, el decreto autoriza ampliar 200 metros el "anillo de contención verde" del Gran Área Metropolitana (GAM), lo que abre un total de 54562000 metros cuadrados para la urbanización, ampliando la posibilidad de construir hasta en un $50 \%$ del área total de las fincas. Sin embargo, esta resolución conlleva otro tema de fondo mucho más sensible que se relaciona directamente con la política pública en materia de ordenamiento territorial para los próximos treinta años y el grado de impacto que pueda tener sobre la protección de los recursos naturales. El decreto pone sobre la mesa el tema ambiental versus el crecimiento urbanístico, mostrando de nuevo la interrogante ¿cuánto más podemos crecer sin comprometer nuestro medio ambiente? Visualizando una temática transversal mucho más profunda ¿quién dictamina hasta dónde puede llegar el desarrollo territorial y urbanístico de nuestra GAM?

\section{ACTORES INVOLUCRADOS}

El decreto es presentado por el Ministerio de Vivienda y Asentamientos Humanos (MIVAH), el Instituto Nacional de Vivienda y Urbanismo (INVU), el Ministerio de Ambiente, Energía y Telecomunicaciones (MINAET) y la Presidencia de la República. Sin embargo, en declaraciones a medios de comunicación nacional sólo se han manifestado los jerarcas del MIVAH y el INVU, este último ha sido el mayor propulsor de la medida. En la Ley de Planificación Urbana de 1987 se establece en su artículo 2, capítulo segundo que la Dirección de Urbanismo del INVU llevará a cabo un control jurídico y técnico de los planes reguladores locales en tutela de los intereses nacionales $y$ regionales relativos al desarrollo urbano ${ }^{3}$.

Como un segundo actor se encuentra el MIVAH, al tener como parte de sus políticas

$3 \quad$ Esta competencia de control ha sido avalada por la Sala Constitucional en su sentencia número 544599 interpretando que, con ello, no se quebranta el ejercicio autónomo de una competencia municipal porque la función del INVU es precisamente asegurar que los planes reguladores se acomoden a los intereses urbanísticos nacionales y regionales. 
el desarrollo de la planificación urbana para el ordenamiento territorial y la definición de lineamientos e instrumentos que permitan el desarrollo de asentamientos humanos equitativos y sostenibles. De acuerdo con Mata, (La Nación, 2010) entre sus declaraciones a medios de comunicación apoyando la medida, la Ministra Clara Zomer negó categóricamente que el decreto genere una afectación en el ambiente, pues sostiene que la normativa respeta el protocolo de la Secretaría Técnica Nacional Ambiental (SETENA).

No obstante, más allá de que este Ministerio ha apoyado la medida, como lo indica (Román, 2010) dentro de la institución no se ha elaborado ninguna notificación oficial con respecto al documento ni se ha colaborado con la construcción de algún tipo de criterio técnico que lo sustente.

Finalmente, dentro de los considerandos del documento aparece la SETENA como encargado de velar por la viabilidad ambiental que facultaría las construcciones en esas zonas, sin embargo Sonia Espinoza, Directora General de la institución, afirmó al periódico La Nación (Mata, 2010) que dicha entidad no tuvo participación en el decreto y afirmó desconocer los alcances de este.

Sin embargo, dentro de los considerandos VI, VII, VIII del documento en estudio claramente se expresa que la medida utilizará la viabilidad ambiental dada por la SETENA para asignar las construcciones.

[que] Mediante Resolución 1308-2009-setena, el Ministerio de Ambiente, Energía y Telecomunicaciones, otorga estudio técnico de viabilidad ambiental al plan PRUGAM, lo que permite al Estado basarse en estos estudios técnicos para mantener la protección a un ambiente sano y ecológicamente equilibrado.

Por otra parte, se encuentra la posición del proyecto Planificación Regional y Urbana de la Gran Área Metropolitana (PRUGRAM). A partir de la creación del Consejo Nacional de Planificación Urbana (CNPU) y la Secretaría del
Plan Nacional de Desarrollo Urbano (PNDU) ${ }^{4}$ se impulsa la elaboración del Plan Nacional de Desarrollo Urbano, el cual se planteó por etapas. Su fase II consiste en actualizar el Plan GAM, así como los Planes Reguladores de los 31 municipios que la componen. Este proyecto (Brenes, 2010) pasa a denominarse Proyecto de Planificación Regional y Urbana del Valle Central de Costa Rica (GAM) o Proyecto PRUGAM. Propiamente (Brenes, 2010) la posición del PRUGRAM con respecto a este decreto refiere a cómo puede contraponerse este a las herramientas construidas por el programa, particularmente el componente de la viabilidad ambiental y la colaboración con las municipalidades de la región para construir sus planes reguladores y que estos a su vez incluyan esta viabilidad.

De acuerdo con Reglamento general sobre los procedimientos de evaluación de impacto ambiental de SETENA, la Evaluación Ambiental Estratégica tiene como objetivo integrar la variable de impacto ambiental a la planificación del desarrollo económico del país. Se aplica a los planes, programas y políticas de desarrollo nacional, regional y local generados en municipios, cuencas hidrogeográficas y regiones específicas; $y$ cuyo fin sea el planteamiento del uso de suelo, el desarrollo de infraestructura (urbana, vial, portuaria, energética, turística, entre otras).

Son estas dos herramientas las que principalmente fundamentan la posición del PRUGRAM en relación con este decreto, ya que este proyecto construye la viabilidad ambiental utilizando toda la legislación existente para ser usada en todos los planes nacionales, regionales y locales; la cual plantea que dentro del área de contención es posible construir en las zonas de alta fragilidad hasta un $20 \%$ mientras en las zonas de fragilidad moderada hasta un $70 \%$. Esto se contrapone al 50\% de posibilidad de construcción que plantea el decreto.

4 El Gobierno de la República emitió el Decreto Ejecutivo 28937 MOPT-MIVAH-MINAE el 9 de octubre de 2000, en el cual se crea el Consejo Nacional de Planificación Urbana (CNPU), y la Secretaría Técnica del Plan Nacional de Desarrollo Urbano. 
Esta evaluación ambiental fue trabajada por la SETENA y PRUGRAM hasta que en la resolución 1308-2009-SETENA realizada el 08 de junio de 2009, se acuerda otorgar la Viabilidad Ambiental al Plan PRUGAM 2008-2030. Esto implica que este instrumento debe utilizarse en todo plan local, regional, nacional o maestro previo a su autorización final.

De esta forma se indica:

... de conformidad con la legislación vigente, se ha realizado una revisión puntual de la integración de la variable ambiental en el Plan PRUGAM 2008-2030, conforme al "Procedimiento Técnico para la Introducción de la Variable Ambiental en los Planes Reguladores u otra Planificación de Uso del Suelo", Decreto Ejecutivo 32967-MINAE. Revisión que de acuerdo con la metodología, cumple con lo solicitado por esta secretaría.

Asimismo, el Reglamento General sobre Procedimientos de Evaluación de Impacto Ambiental, publicado mediante Decreto Ejecutivo 31849-MINAES- MOPT-MAG-MEIC, indica en su artículo 67 sobre la Integración de variable ambiental en los Planes Reguladores así como en su artículo 19 de la Ley Orgánica del Ambiente señala que: "Las resoluciones de la Secretaría Técnica Nacional Ambiental deberán ser fundadas y razonadas. Serán obligatorias tanto para los particulares como para los entes y organismos públicos".

Junto al PRUGRAM varios movimientos ambientalistas se han dado a la tarea de repudiar esta iniciativa por su alto impacto en la zonas de recarga acuífera, así como en las zonas de riesgo, uno de estos grupos es el denominado Llamado urgente, que de acuerdo con Rodríguez (Semanario Costa Rica Hoy, 2010) ha arrojado los siguientes datos en diversos medios de comunicación que ejemplifican los alcances ambientales de la medida. Las áreas de muy alta $y$ alta fragilidad ambiental dentro de esa franja de tierra que cubre 25 cantones, es de 4118 hectáreas, es decir, el 75,5\% del total. Estas no son aptas para el desarrollo urbano intenso, son aptas para la actividad agrícola o la conservación ambiental, o bien, algunas de ellas, para un desarrollo urbano controlado y condicionado. Mientras las áreas de moderada fragilidad, representan apenas $1330 \mathrm{Ha}$, es decir, sólo el 24,5 \% del total de la franja.

Además, el abogado ambientalista Álvaro Sagot y el físico José Alberto Brenes presentaron una acción de inconstitucionalidad, esta acción alega que el decreto violenta la protección especial que emana de la norma 21, 50 y 89 de la Constitución Política.

\section{CONFLICTO CON EL DECRETO}

Una vez planteada la institucionalidad que rodea el ordenamiento territorial y urbanístico, es posible visualizar las contradicciones existentes en el decreto. Dentro de los considerandos del mismo se mencionan una serie de herramientas políticas e institucionales que, según el ordenamiento jurídico existente, son la base que rige la planificación territorial; sin embargo, estas disposiciones llegan a contraponerse a lo dictado en el artículo.

La Secretaría del Plan Nacional de Desarrollo Urbano, por medio del proyecto PRUGAM, se dio a la tarea de establecer un Plan de Desarrollo Urbano para el Gran Área Metropolitana de largo plazo, el cual tiene la tarea de apoyar al INVU en la definición de la política de ordenamiento urbanístico del país. Este proyecto tiene dos componentes, uno la viabilidad urbanística cuya aprobación corresponde al INVU de acuerdo con los artículos 2 y 3 de la Ley de Planificación Urbana. Y en el segundo componente se encuentra la viabilidad ambiental que corresponde a los Indicadores de Fragilidad Ambiental (IFAs) creados por PRUGAM, así como la Evaluación Ambiental Estratégica correspondiente al Decreto 31849-MINAE-MS-MOPT-MAG-MEIC. Esta viabilidad ambiental fue acogida por la SETENA en la resolución 1308-2009-SETENA Plan Regulador PRUGAM 2008-030 expediente número EAE-0142008-SETENA, la cual indica que la metodología desarrollada por PRUGAM debe ser incorporada en cualquier propuesta local, regional, nacional o maestro y es de acatamiento obligatorio. 
De acuerdo con Eduardo Brenes, ex director del PRUGAM, esta metodología se encuentra incorporada al menos en 12 municipalidades.

Una vez aclarado los elementos que rodean el decreto a continuación se detallan las áreas polémicas:

1. Actualmente el INVU no ha iniciado el trámite de aprobación del Plan PRUGAM, esto conlleva a que en este momento el plan que se encuentra vigente es el de la Ley de Planificación Urbana de 1982. Esto da la competencia a esta institución para evaluar los planes reguladores que se presenten.

El plan de 1982 no incluye la viabilidad ambiental, tal y como se presenta en el Reglamento de Desarrollo Sostenible de SETENA. En este sentido el decreto, al mencionar en el considerando numero VI este instrumento, está incurriendo en el error de confundir planes y normativas. Es decir, la normativa vigente no incluye la viabilidad ambiental, sino que se incluye en el plan PRUGAM, el cual todavía no ha sido aprobado, por lo que se trata de aplicar una medida en un contexto al cual no le corresponde dado que PRUGAM tiene estudios más actualizados por lo que no se puede trasladar resoluciones. En este sentido es importante mencionar que para que el plan PRUGAM funcione adecuadamente $y$ sea implementado correctamente, es necesaria la coexistencia de tanto la viabilidad ambiental como la urbanística, de forma tal que, para que la actual viabilidad ambiental pueda ir mejorando con el tiempo, requiere la articulación del plan urbanístico en cada una de sus áreas.

2. Siguiendo con lo indicado en el considerando número VI, solo se menciona la resolución 1308-2009-SETENA, obviando la existencia de dos resoluciones más, la 1532-2009-SETENA en la cual se establecen lineamientos ambientales y la resolución 2748-2009-SETENA, en donde se aprueba el Reglamento de Desarrollo
Sostenible que forma parte de la viabilidad ambiental. Además, se menciona la resolución 1308-2009-SETENA como si fuese un estudio técnico de viabilidad ambiental, y no como una resolución, esto representa un problema debido a que se utilizó como el criterio técnico base para avalar la necesidad de ampliar el anillo de contención, arguyendo también la viabilidad ambiental, cuando esta resolución no es criterio suficiente para justificar la ampliación, además de que la misma resolución indica la existencia de sectores con muy alta fragilidad ambiental que deben tratarse bajo consideraciones especiales de protección.

3. Del considerando VI también se deriva que se consultaron los estudios técnicos del plan PRUGAM, esto es refutable desde tres puntos de vista. Primero, el decreto divide los terrenos en zonas de alta fragilidad $y$ fragilidad moderada, sin embargo, la resolución de SETENA 2748-2009 que refiere al Reglamento de Desarrollo Sostenible establece una serie más amplia de subclases para las categorías de los IFas. Por ejemplo, en las zonas I-A e I-C la ocupación humana es de muy baja intensidad, por lo que se recomienda no superar el $10 \%$ de cobertura. En las zonas II-B la ocupación humana es de baja y moderada intensidad y no debe superar el 40\%. En zonas III-A la ocupación humana es de alta y muy alta densidad pero con porcentajes que no superen el 50\%. Esto lleva al segundo problema que se refiere a los porcentajes utilizados en el decreto, los cuales no tienen relación con lo establecido por el PRUGAM, es decir el decreto establece hasta un $50 \%$ para construcción, sin embargo el PRUGAM indica que es posible construir en las zonas de alta fragilidad hasta un $20 \%$, mientras en las zonas de fragilidad moderada hasta un $70 \%$. Sobre este tema, el INvU afirma que ese $50 \%$ (correspondiente a "una cuadra" en el vocabulario urbanístico) siempre se ha mantenido como un espacio flexible, 
dado que es comprensible que el proceso de urbanización sea constante y dinámico; lo que sí indican es que el otro 50\% no se puede tocar bajo ninguna circunstancia. Por último, no queda claro cuál anillo de contención se seleccionó para el decreto, debido a que existen tres versiones: la original del Plan GAM de 1982, la versión de 1997 del INVU, en la cual se mantiene el anillo de 1982, pero se adicionan pequeños anillos de contención urbana, que en su mayoría coinciden con cabeceras de cantón; y la versión del PRUGAM. Por otra parte, los estudios técnicos del PRUGAM para determinar el anillo de contención fueron determinados por medio de sistemas de información geográfica, lo que vuelve más específico y acertado este límite imaginario, en contraposición del anillo de 1982 que no contó con este tipo de tecnología.

4. Finalmente, no queda claro qué sucede con las zonas de amortiguamiento o de protección que quedan dentro del anillo de contención ampliado, afectándose sitios como Loma Salitral, Cerros de Escazú y Cerros de la Carpintera, entre otros. De acuerdo con funcionarios del INVU, las zonas protegidas, zonas de amortiguamiento $y$ acuíferos no podrán ser afectadas por el decreto.

\section{CONSIDERACIONES FINALES}

Con base en la documentación analizada $y$ las entrevistas realizadas, es posible concluir que el actual decreto adolece de una grave descoordinación interinstitucional, además de irrespetar una serie de normativas y planes vigentes. Entre los hallazgos más relevantes se encuentran:

1. El decreto carece de algún estudio técnico previo que lo respalde, incluso no sólo utiliza dictámenes técnicos de la SETENA como estudios técnicos, sino que los utiliza de manera incorrecta.

2. La institución encargada de articular las políticas de ordenamiento territorial, en este caso el INVU, no ha realizado una propuesta concreta y completa para generar un plan de ordenamiento regional urbano, $y$ además no ha iniciado el proceso de evaluación del plan PRUGAM para medir sus posibilidades de aplicación política y técnica.

3. Los actores involucrados en el decreto, específicamente SETENA, INVU y MIVAH, no participaron en su elaboración ni en la realización de estudios técnicos que apoyen la medida. Además, aunque la ex-ministra Clara Zomer firmó el decreto, pero a lo interno del Ministerio no se tenía conocimiento sobre él. Lo mismo sucede en el INVU, donde no se participó en la redacción del decreto, por lo que no queda claro ¿cuál institución? y bajo ¿qué criterios se redactó el decreto en cuestión?

4. El decreto, a grandes rasgos, abre un portillo para que los desarrolladores inmobiliarios construyan en estas zonas debido a que, de no aprobarse el plan PRUGAM, continúa vigente el Plan GAM de 1982, en el cual el INvU tiene facultad de toma de decisión en la aprobación de los planes reguladores de las municipalidades, $y$ en este momento sólo once cuentan con planes reguladores con viabilidad ambiental. Esto se sustenta en cuatro argumentos:

a. Entre 1970-1973 se elaboraron propuestas separadas de planes cantonales para nueve de los diez cantones del Área Metropolitana; sin embargo, únicamente la Municipalidad de Montes de Oca cumplió con todos los requisitos para la aprobación final y publicación en "La Gaceta" del plan de ese cantón. En 1973 el INVU desiste de la idea de planes reguladores para cada uno de los cantones del Área Metropolitana por considerarlos demasiado particulares e inoperantes, por lo que ordenó realizar un estudio total del Área Metropolitana. 
b. El único plan regional urbano dictado al amparo de la Ley de Planificación Urbana (LPU) es el vigente Plan Regional de Desarrollo Urbano "Gran Área Metropolitana” (GAM), aprobado mediante decreto 13 583-vaHOFIPlan. El plan fue elaborado por el INVU en aplicación de lo dispuesto en el transitorio II de la LPU, según la directriz número 12 590-P, que le permite al instituto dictar planes reguladores, delimitar distritos urbanos y demás áreas sujetas a control urbanístico, “... en tanto las municipalidades no hubieran promulgado en la respectiva materia".

c. La Sala IV señaló en la sentencia 6706-93, que

III).- Dentro de lo que puede denominarse la organización administrativa del urbanismo en nuestro país, la Dirección de Urbanismo -adscrita al Instituto Nacional de Vivienda y Urbanismo- y la Oficina de Planificación (hoy día Ministerio de Planificación y Política Económica) son los órganos encargados de elaborar el Plan Nacional de Desarrollo Urbano, a través del cual se fijan las políticas generales sobre el crecimiento $y$ desarrollo de las áreas urbanas.

d. El Transitorio II de la Ley de Planificación Urbana que textualmente dice:

El Instituto dictará las normas de desarrollo relativas a las materias a que se refiere el Art. 212 de esta ley. Podrá, además, confeccionar los planes reguladores y delimitar los distritos urbanos y demás áreas sujetas a control urbanístico, en tanto las municipalidades no hubieren promulgado, en la respectiva materia, o parte de ella, sus propias disposiciones locales con ajuste a esta ley. Los preceptos y reglamentos que dicte el Instituto regirán en los territorios jurisdiccionales o en la parte de ellos que las normas señalen a partir de su publicación en el Diario Oficial, [dejando las Municipalidades al arbitrio estatal el desarrollo urbano de sus centros poblados, o a la iniciativa privada las propuestas de ordenamiento en las zonas costeras].

5. El INVU no tiene objeciones sobre la posibilidad que establece el decreto para construir en ciertas zonas del anillo de contención, dado que funcionarios afirman que la medida no los tomó por sorpresa debido a que el aumento y disminución del anillo de contención es un tema que ha sucedido varias veces desde 1983. Sin embargo, (Acuña, 2010) sí consideran que la forma en la que se incorporó el tema ambiental es bastante "tímida e incipiente" ya que no establece criterios técnicos específicos que puedan evitar portillos para construir en lugares no aptos. Además, consideran que el decreto se escribió "en un escritorio" porque asume que todas las áreas son iguales y no toma en consideración zonas donde los 200 metros son despeñaderos, laderas u otro tipo de zona no apta para la construcción. Por otro lado, sí reconocen la importancia del decreto debido a que dentro de esos 200 metros ya existen construcciones, $y$ el reacomodo de familias o comunidades enteras sería un proceso social altamente conflictivo y no deseable.

6. Contrario al argumento del INVU sobre la necesidad de nuevos espacios de construcción debido al crecimiento de la población y el hecho de que contenga regulaciones para proteger el medioambiente, PRUGAM y PRODUS, según declaraciones y estudios advierten que el crecimiento poblacional del GAM no requiere de la ampliación de construcciones, ya que, con el uso adecuado de la infraestructura y terrenos existentes, $y$ con planificación urbana moderna, la futura población de la 
GAM tiene espacio suficiente para su crecimiento. Además, San José todavía tiene niveles de despoblación que deben ser aprovechados.

7. Es necesario aprovechar y magnificar los espacios urbanos, no sólo por el aprovechamiento del espacio per se, sino por los costos en provisión de servicios que le representan al país el hecho que la urbanización crezca desmedida y desordenadamente. Para ello es necesario replantear el modelo de vivienda de una familia/una casa, $y$ aprovechar los espacios hacia arriba. De acuerdo con José Gabriel Román funcionario del MIVAH, si se diseñan barrios con servicios completos (áreas verdes, áreas comunales/sociales, espacios de estudio y esparcimiento, etc.), se puede crear un nuevo modelo urbano de aprovechamiento de espacios antes no utilizados. Además, es urgente modernizar el sistema de transporte del país.

8. De acuerdo con varios expertos en el tema, el proyecto PRUGAm tiene excelentes elementos técnicos, así como buenos planteamientos urbanos, sin embargo, no tomó en consideración el factor político que al final puede ser el que defina su éxito o fracaso. Por otra parte, existe la confusión de que el PRUGAM es un programa definitivo, cuando realmente es un documento final que sirve como base para iniciar una discusión sobre cómo debe organizarse la GAM de acuerdo con elementos sociales, económicos y políticos, sin olvidar que ya es un texto de referencia $y$ probablemente lo será por muchos años más dada su complejidad. De acuerdo con el señor José Gabriel Román, funcionario del MIVAH "los productos de PRUGAM son técnicamente muy valiosos pero políticamente complejos. Las posibilidades reales de transformar los documentos en herramientas reales de gestión institucional son discutibles".

9. Aunque (Morúa, 2010) en el INVU se afirma que, de aprobarse el decreto, absolutamente todo seguiría igual, no se afectarían zonas protegidas y el proyecto PRUGAM seguiría su curso natural; sin embargo, existen quienes no están de acuerdo con esta afirmación. Por lo que también es necesario preguntarse cómo podría afectar la aprobación del decreto las relaciones del país con la Unión Europea, especialmente por la amplitud del proyecto.

10. Por otro lado, los procesos de planificación se conciben de lo particular a lo general. Se asume que la suma de los Planes Reguladores define las políticas regionales y nacionales. En consecuencia, no existen políticas generales ni regionales, y los Planes Reguladores realizados hasta el momento no han mostrado una suficiente capacidad para orientar el desarrollo urbano. Además de la Dirección Urbanismo del INVU y las municipalidades, existen una serie de leyes que la dan a múltiples instituciones, injerencia en estos campos.

11. Cabe señalar que el Poder Ejecutivo ha efectuado dos intentos previos para abrir el "anillo de contención". La Sala Constitucional anuló en 2009 el Decreto 33757-MP-MINAH-MINAE que reformaba el Plan Regional de Desarrollo Urbano del Gran Área Metropolitana, al declarar con lugar la acción de inconstitucionalidad número 07-11509-0007-co.

Este decreto adicionaba un inciso al artículo 3 del Decreto Ejecutivo 25902-MIVAH-MP-MINAE, cuyo texto dice:

(...) 3.7 Las fincas por cuyos terrenos se definió el límite de contención urbana o que estén próximos con este a no más de cien metros, podrán exceder el uso urbano dentro del área de protección en un porcentaje no mayor del $50 \%$ del área total de la finca o hasta doscientos metros del límite, cualquiera que fuese menor $(. .$.

La Sala, en su sentencia 3684-2009, afirmó que este Decreto viola el derecho fundamental a un ambiente sano y ecológicamente equilibrado al permitir 
el desarrollo urbanístico en el anillo o límite de contención del Gran Área Metropolitana, afectando las áreas de recarga acuífera, el patrimonio forestal $y$ el paisaje, motivos que sustentan su protección especial. Además consideró que este decreto desconocía la obligación del Estado de tutelar debidamente el ambiente y los elementos que lo conforman. Esto muestra un fuerte interés de parte del Ejecutivo de abrir este anillo a pesar de que la Sala ya se ha pronunciado sobre el tema.

12. La Constitución en su artículo 169 y la Ley de Planificación Urbana en su artículo 15 reconocen "la competencia y autoridad de los gobiernos municipales para planificar y controlar el desarrollo urbano, dentro de los límites de su territorio jurisdiccional" sin embargo, es una realidad que las municipalidades carecen de medios técnicos y financieros para hacerle frente a una responsabilidad tan grande como esta.

En este sentido muchos de los requisitos que se establecen para generar un plan regulador no son acatados por las municipalidades. De acuerdo con el documento "La institucionalidad del Plan Nacional de Desarrollo Urbano", se indica que no se plantea una política de desarrollo del área, no se realizan proyecciones de población, no se practican estudios de la circulación, no se incluye la destinación de áreas para servicios comunales o servicios públicos, no se practica regularmente un análisis de ubicación de actividades y finalmente, no se practican ejercicios de vivienda $y$ renovación urbanas.

Si bien las municipalidades poseen autonomía dentro de su territorio, es importante contar con planes regionales que brinden a los gobiernos locales herramientas adecuadas de gestión territorial, $y$ propicien el mejoramiento del desarrollo y evaluación de los planes reguladores que se construyan. Sin embargo, actualmente al no contar con un plan nacional actualizado y moderno, la tarea de los gobiernos locales en relación con el ordenamiento territorial se vuelve más difícil.

13. La iniciativa Paz con la Naturaleza cuenta con una política de ordenamiento territorial, la cual establece claramente la necesidad de un plan nacional en este tema y de articular las políticas vigentes con las nuevas iniciativas que se están desarrollando. No obstante, a la luz de este decreto se puede establecer una contradicción entre ambas iniciativas; según lo analizado en este artículo ambas pueden llegar a contraponerse entre sí, a pesar de que son fomentadas por el Ejecutivo de esta Administración. En este sentido la Secretaría Técnica de Planificación Urbana junto a la política de ordenamiento territorial deberían ir marcando el norte sobre el desarrollo urbanístico de este país como parte de sus objetivos de creación. La Secretaría no presenta el decreto, a pesar de que esta debe ser el órgano articulador del nuevo Plan de Desarrollo Urbano y a la vez un ente capaz de poner en práctica las premisas de la política de ordenamiento territorial.

14. Dada la ineficiencia institucional, las duplicidades de funciones $y$ el desfase de normativas, se ha fallado en el desarrollo urbano del país, el cual ha sido completamente caótico, de poca calidad $y$ perjudicial para el medioambiente.

\section{BIBLIOGRAFÍA}

Brenes, E. Presentación Plan PRUGAM. En: <http: //www.prugam.go.cr/ presentacion_director_ministro.htm> [Consultado el 11 de abril de 2010].

Decreto Ejecutivo 28937 MOPT-MIVAH-MINAE.

Decreto 31777-MIVAH-MIDEPLAN. 
Decreto Ejecutivo 34043-MINAE.

Decreto Ejecutivo 35748-MP-MINAET-MIVAH.

El Financiero. "UCR rinde informe sobre conflictivo acueducto en Sardinal". En: <http://www.elfinancierocr. com/ef_archivo/2010/marzo/07/ economia2290882.html> [Consultado el 30 de marzo de 2010].

Estado de la Nación, Costa Rica. XIII Informe del Estado de la Nación. En: <http:// www.estadonacion.or.cr/Info2007/ Paginas/armonia.htm $>$ [Consultado el 30 de marzo de 2010].

Estado de la Nación, Costa Rica. XIV Informe del Estado de la Nación. En: <http:// www.estadonacion.or.cr/Info2008/ Paginas/armonia.html> [Consultado el 30 de marzo de 2010].

Estado de la Nación, Costa Rica. XV Informe del Estado de la Nación. En: <http:// www.estadonacion.or.cr/pdf/Resumen_ armonia.pdf $>$ [Consultado el 30 de marzo de 2010].

Jiménez, J. “¿Mare Nostrum?”. Periódico La Nación. En: <http://www.nacion. com/2010-03-03/0pinion/Foro/ Opinion2287630.aspx>

Asamblea Legislativa, Costa Rica. Ley de Planificación Urbana 4240.

Revista Ambientico. Manifiesto público en defensa de la naturaleza. En: <http:// www.ambientico.una.ac.cr/eventos/ MANIFIESTO.pdf $>$ [Consultado el 7 de abril del 2010].

Mata, A. "Decreto permite construir en áreas de protección ambiental". Periódico La Nación. Jueves 25 de febrero de 2010.
Ministerio de Ambiente, Energía y Telecomunicaciones. Secretaría Técnica Nacional Ambiental (SETENA). Resolución 1308-2009-SETENA. Plan Regulador PRUGRAM 2008-2030. Expediente número eae-014-2008-SETENA. 9 de junio de 2009.

Ministerio de Vivienda y Asentamientos Humanos. Politicas Institucionales. En: <http://www.mivah.go.cr/Politicas Institucionales.shtml> [Consultado el 13 de abril de 2010].

Organización Llamado Urgente por el País. "Graves desaciertos ambientales de la Administración Arias 2006-2010". Comunicado de prensa. 9 de abril de 2010.

Ministerio de Planificación Nacional y Política Económica, Costa Rica. Plan Nacional de Desarrollo (PND) 2006-2010.

MINAE, MS, MOPT, MAG, MEIC. Reglamento General sobre los Procedimientos de Evaluación Impacto Ambiental. Decreto 31849-MINAE-MS-MOPT-MAG-MEIC. 2004.

SETENA. Resolución 1308-2009.

Rodríguez, M. "Denuncian que 75\% de terrenos "liberados" en el Gran Área Metropolitana no son aptos para urbanizar". Semanario Costa Rica Hoy. Martes 13 de abril, 2010.

Semanario Universidad. "Buscan cómo frenar caótico desarrollo costero". En: <http:// www.semanario.ucr.ac.cr/index.php/ mainmenu-pais/150-caotico-desarrollocostero.html> [Consultado el 30 de marzo de 2010].

Sagot, Á.; Brenes, J. Acción de inconstitucionalidad. Palmares 1 de marzo de 2010.

Sala Constitucional. "Sentencia 3684-2009”.

Sala Constitucional. "Sentencia 5445-99". 


\section{ENTREVISTAS}

Acuña, Luis Fernando. Instituto Nacional de Vivienda y Urbanismo (INVU). 15 de abril de 2010.

Brenes, Eduardo. Programa Regional Urbano para el Gran Área Metropolitana (PRUGRAM). 8 de abril de 2010.

Mora, José Ramón. Departamento de Evaluación, SETENA. 07 de abril de 2010.
Morúa, Meyer. Instituto Nacional de Vivienda y Urbanismo (INVU). 15 de abril de 2010.

Pujol, Rosendo. Programa de Investigación en Desarrollo Urbano Sostenible, Escuela de Ingeniería Civil, Universidad de Costa Rica. 14 de abril de 2010 .

Román, José Gabriel. Ministerio de Vivienda y Asentamientos Humanos (MIVAH). 8 de abril de 2010. 